\title{
Many-body effects and matrix inversion in low-Reynolds-number hydrodynamics
}

\author{
Kengo Ichiki ${ }^{\mathrm{a})}$ \\ Graduate School of Human and Environmental Studies, Kyoto University, Kyoto 606-8501, Japan \\ John F. Brady \\ Division of Chemistry and Chemical Engineering, California Institute of Technology, Pasadena, \\ California 91125
}

(Received 5 August 1999; accepted 3 October 2000)

\begin{abstract}
It is shown that the method of reflections in resistance form (with truncated multipoles) is one of many possible iterative methods to obtain the inverse of the mobility matrix (with the same truncation) in low-Reynolds-number hydrodynamics. Although the method of reflections in the mobility form is guaranteed to converge, it is found that in the resistance form the method may fail to converge. This breakdown is overcome by conjugate-gradient-type iterative methods, and the implications of the iterative method for low-Reynolds-number hydrodynamics are discussed.
\end{abstract}

(C) 2001 American Institute of Physics. [DOI: 10.1063/1.1331320]

In low-Reynolds-number hydrodynamics, the interaction among particles is completely characterized by either the resistance or the mobility matrix. ${ }^{1,2}$ The former gives the forces in terms of the velocities, and the latter gives the velocities in terms of the forces; they are inverses of each other. In the Stokesian dynamics method to simulate particle motion at low Reynolds numbers, an approximate pair-wise mobility matrix is constructed and inverted as a way to take into account long-range, many-body hydrodynamic interactions. ${ }^{3}$ Durlofsky et al. ${ }^{3}$ showed for a pair of particles that this inversion of the pair-wise mobility matrix is equivalent to a summation of an infinite series of reflected resistance interactions. The method of reflections is an iterative method to solve for the hydrodynamic interactions among particles ${ }^{2}$ and thus is a way to take into account many-body interactions. While the convergence of the method of reflections has been proven for the mobility problem, ${ }^{4}$ the convergence for the resistance problem is an open question. We shall see that for more than two particles the method of reflections in the resistance form may fail to converge, which has important implications for Stokesian dynamics-like algorithms that wish to bypass the computationally costly mobility matrix inversion and still incorporate many-body effects. However, the method of reflections is only one of many iterative methods that can be used to invert the mobility matrix, and other iterative techniques can be used to insure convergence.

We briefly summarize the method of reflections with truncated multipoles in resistance form and then clarify the relation between the method of reflections and the inversion of the mobility matrix. Next we examine the convergence of the method of reflections for many-body problems in detail and show that the method may fail to converge. It is also shown that conjugate-gradient-type iterative methods can overcome the convergence limitations. We conclude with a

${ }^{a)}$ Electronic mail: ichiki@kona.jinkan.kyoto-u.ac.jp discussion of the implications of these findings for particle simulation methods.

At low Reynolds numbers with a rigid spherical particle $\alpha$ placed at $\mathbf{x}_{\alpha}$, the velocity field at $\mathbf{x}$ can be written as

$$
\mathbf{u}(\mathbf{x})=\frac{1}{8 \pi \mu}\left(1+\frac{a^{2}}{6} \nabla^{2}\right) \mathrm{J}\left(\mathbf{x}-\mathbf{x}_{\alpha}\right) \cdot \mathbf{F}_{\alpha}+\cdots,
$$

where $\mathbf{F}_{\alpha}$ is the force exerted by particle $\alpha$ on the fluid, $\mathrm{J}(\mathbf{r})$ is the Oseen tensor,

$$
\mathrm{J}(\mathbf{r})=\frac{1}{r}\left(\mathrm{I}+\frac{\mathbf{r r}}{r^{2}}\right)
$$

and $\mathrm{I}$ is the unit tensor. The viscosity of the fluid is $\mu$. For simplicity, we only discuss in detail the truncation of (1) at the level of the zeroth moment-the force, which gives the so-called F-version in Stokesian dynamics; that is, we neglect the torque and higher order force moments. The extension to include higher moments ${ }^{3,5}$ is straightforward.

The above is a mobility representation giving the velocity in terms of the force. To construct a resistance formforces in terms of velocities-we make use of Faxén's law ${ }^{2}$ to write

$$
\mathbf{F}_{\alpha}=6 \pi \mu a\left[\mathbf{U}_{\alpha}-\left(1+\frac{a^{2}}{6} \nabla^{2}\right) \mathbf{u}^{\prime}\left(\mathbf{x}_{\alpha}\right)\right],
$$

where $\mathbf{U}_{\alpha}$ is the translational velocity of particle $\alpha$ with center at $\mathbf{x}_{\alpha}$, and $\mathbf{u}^{\prime}(\mathbf{x})$ is the velocity field in which particle $\alpha$ is immersed-due to an imposed flow or caused by other particles. Faxén's law (2) is exact provided that $\mathbf{u}^{\prime}$ is the exact solution to the Stokes flow problem subject to the proper boundary conditions on the surfaces of all the particles. In general, we do not know the exact form of $\mathbf{u}^{\prime}$, which would be a complete solution to the many-body problem, but rather build it up in an iterative manner: first ap- 
proximating the velocity field as that due to noninteracting particles, e.g., (1), and then correcting the force density of particle $\alpha$ by (2), and so on.

Consider two particles: neglecting all interactions between these particles we have for the force on particle $\alpha$ translating with velocity $\mathbf{U}_{\alpha}$

$$
\mathbf{F}_{\alpha}^{(0)}=6 \pi \mu a \mathbf{U}_{\alpha},
$$

where the superscript $(0)$ denotes the zeroth-order reflection. The force on a particle now causes a velocity disturbance, which can be expressed by the multipole expansion (1) as

$$
\mathbf{u}^{(0)}(\mathbf{x})=\frac{1}{8 \pi \mu}\left(1+\frac{a^{2}}{6} \nabla^{2}\right) \mathrm{J}\left(\mathbf{x}-\mathbf{x}_{\alpha}\right) \cdot \mathbf{F}_{\alpha}^{(0)} .
$$

Substituting this disturbance flow into Faxén's law (2), we obtain the force on particle $\beta$ due to particle $\alpha$ with the first-order reflection correction:

$$
\mathbf{F}_{\beta}^{(1)}=6 \pi \mu a\left[\mathbf{U}_{\beta}-\frac{1}{8 \pi \mu}\left(1+\frac{a^{2}}{6} \nabla^{2}\right)^{2} \mathrm{~J}\left(\mathbf{x}_{\beta}-\mathbf{x}_{\alpha}\right) \cdot \mathbf{F}_{\alpha}^{(0)}\right] .
$$

This procedure can be repeated, with the $i$ th order reflection written in a recurrent equation as

$\mathbf{F}_{\beta}^{(i)}=6 \pi \mu a\left[\mathbf{U}_{\beta}-\frac{1}{8 \pi \mu}\left(1+\frac{a^{2}}{6} \nabla^{2}\right)^{2} \mathrm{~J}\left(\mathbf{x}_{\beta}-\mathbf{x}_{\alpha}\right) \cdot \mathbf{F}_{\alpha}^{(i-1)}\right]$.

It is easy to extend this procedure to the $N$-body problem by superposing disturbances by the other particles.

We can summarize the method of reflections of the F-version in a matrix form as

$\mathbf{F}^{(i)}=\mathcal{I} \cdot\left[\mathbf{U}-\tilde{\mathcal{M}} \cdot \mathbf{F}^{(i-1)}\right]=\mathcal{I} \cdot\left(\mathrm{I}+\sum_{k=1}^{i}[-\tilde{\mathcal{M}} \cdot \mathcal{I}]^{k}\right) \cdot \mathbf{U}$,

where $\mathcal{I}$ and $\tilde{\mathcal{M}}$ are defined by

$$
\mathcal{I}=6 \pi \mu a \mid, \quad \tilde{\mathcal{M}}=\left[\begin{array}{cccc}
0 & \mathrm{M}_{12}^{\mathrm{RP}} & \cdots & \mathrm{M}_{1 N}^{\mathrm{RP}} \\
\mathrm{M}_{21}^{\mathrm{RP}} & 0 & \cdots & \mathrm{M}_{2 N}^{\mathrm{RP}} \\
\vdots & \vdots & \ddots & \vdots \\
\mathrm{M}_{N 1}^{\mathrm{RP}} & \mathrm{M}_{N 2}^{\mathrm{RP}} & \cdots & 0
\end{array}\right],
$$

and $\mathrm{M}_{\alpha \beta}^{\mathrm{RP}}$ is the Rotne-Prager tensor

$$
\mathrm{M}_{\alpha \beta}^{\mathrm{RP}}=\frac{1}{8 \pi \mu a}\left(1+\frac{a^{2}}{6} \nabla^{2}\right)^{2} \mathrm{~J}\left(\mathbf{x}_{\alpha}-\mathbf{x}_{\beta}\right) .
$$

In (4) and the following, vectors without particle index are defined as

$$
\mathbf{F}^{(i)}=\left[\begin{array}{c}
\mathbf{F}_{1}^{(i)} \\
\vdots \\
\mathbf{F}_{N}^{(i)}
\end{array}\right], \quad \mathbf{U}=\left[\begin{array}{c}
\mathbf{U}_{1} \\
\vdots \\
\mathbf{U}_{N}
\end{array}\right],
$$

and the power of tensor in (4) means that for a tensor A

$$
\left(A^{k}\right)_{i j}=A_{i l_{1}} A_{l_{1} l_{2}} \cdots A_{l_{k-1} j},
$$

where we have adopted the Einstein summation convention with the repeated indices summed.

It should be noted that the tensor $\tilde{\mathcal{M}}$ is formed by superposition of only two-body effects through the multipole expansion; however, the force $\mathbf{F}^{(i)}$ in (4) has reflections up to $i$ th order among all particles in the system-it includes many-body interactions. In the usual application of the method of reflections one not only takes into account contributions of the reflections but also revises the base solution-in our case, this corresponds to taking into account higher moments in the multipole expansion in (3). In (4) we have considered only force multipoles-the so-called F-version of the Stokesian dynamics method. ${ }^{3,6}$ The velocity disturbance caused by the force on a particle will also induce higher force moments-torques, stresslets, etc.-in a second particle. These can be incorporated into the method of reflections by using the appropriate Faxén law and velocity disturbance for the higher force moments. The FTS version of Stokesian dynamics incorporates the force, torque, and stresslet in this fashion.

Consider the inversion of the matrix $A$ in a linear equation

$$
A \cdot \mathbf{x}=\mathbf{b}
$$

in an iterative manner. First, we split the matrix as

$$
A=A_{1}+A_{2},
$$

and form an iterative procedure

$$
\mathbf{x}=\left(A_{1}\right)^{-1} \cdot\left(\mathbf{b}-A_{2} \cdot \mathbf{x}\right),
$$

giving successively

$$
\begin{aligned}
\mathbf{x}^{(0)} & =\left(\mathrm{A}_{1}\right)^{-1} \cdot \mathbf{b}, \\
\mathbf{x}^{(1)} & =\left(\mathrm{A}_{1}\right)^{-1} \cdot\left[\mathbf{b}-\mathrm{A}_{2} \cdot \mathbf{x}^{(0)}\right], \\
\mathbf{x}^{(i)} & =\left(\mathrm{A}_{1}\right)^{-1} \cdot\left[\mathbf{b}-\mathrm{A}_{2} \cdot \mathbf{x}^{(i-1)}\right] \\
& =\left(\mathrm{A}_{1}\right)^{-1} \cdot\left(\mathrm{I}+\sum_{k=1}^{i}\left[-\mathrm{A}_{2} \cdot\left(\mathrm{A}_{1}\right)^{-1}\right]^{k}\right) \cdot \mathbf{b} .
\end{aligned}
$$

The condition for the convergence of (6) is that the maximum absolute value of the eigenvalues of the iteration matrix " $A_{2} \cdot\left(A_{1}\right)^{-1}$, — called spectral radius-be less than unity. As will be shown in the next section, this criteria is more severe than that of the existence of the inverse-that the determinant of matrix A be non zero. The splitting in (5) is essential to the convergence and its failure means the splitting [and equivalently the initial guess $\mathbf{x}^{(0)}=\left(A_{1}\right)^{-1} \cdot \mathbf{b}$ ] is poor.

Comparing (6) and (4), it is seen that the method of reflections is equivalent to the inversion of the mobility problem

$$
\mathbf{U}=\mathcal{M}^{\infty} \cdot \mathbf{F},
$$

where the mobility matrix is given by

$$
\mathcal{M}^{\infty}=\mathcal{I}^{-1}+\tilde{\mathcal{M}} .
$$

Therefore the method of reflections (4) is an iterative method to invert $\mathcal{M}^{\infty}$ with the splitting (8). This splitting means that the method of reflections is based on the single-body solution ' $\mathcal{I}$. U', and corrects it in each iteration. The iterative method is mathematically equivalent to the Jacobi method ${ }^{7,8}$ where diagonal elements are split, and has the advantage that 


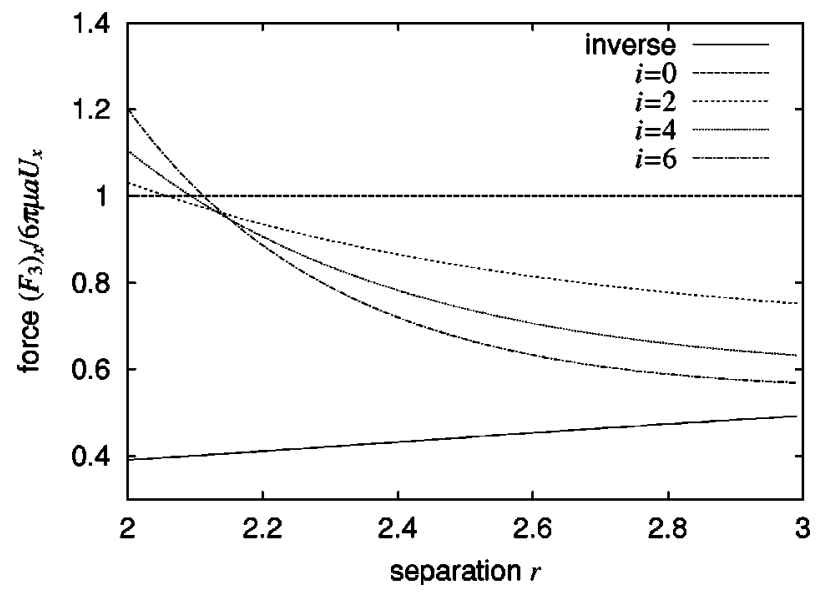

FIG. 1. Force $\left(F_{3}\right)_{x}$ for a three-body problem with a triangular configuration (10) with separation $r$ and velocities (11). The correct value calculated by the inverse of the mobility matrix denoted by "inverse" and the forces with even orders of reflections $i=0,2,4$, and 6 are presented. Odd orders are all lower than the correct value.

the inversion of $\mathcal{I}$ is trivial. If we split with another matrix $\mathrm{A}_{1}$ closer to $\mathcal{M}^{\infty}$, then the convergence may be improved, but at the cost of the evaluation of $\left(A_{1}\right)^{-1}$.

According to the previous analysis, the force calculated by the method of reflections in (4) with an infinite number of reflections $\mathbf{F}^{(i \rightarrow \infty)}$ is equal to the force obtained by the inverse defined by

$$
\mathbf{F}^{\infty}=\left(\mathcal{M}^{\infty}\right)^{-1} \cdot \mathbf{U} .
$$

Therefore, the inversion of the mobility matrix $\mathcal{M}^{\infty}$ takes into account all reflections among all particles in the system. It should be noted that the truncated mobility matrix $\mathcal{M}^{\infty}$ is positive-definite, so that its inverse exists, and the truncation of the multipoles in the method of reflections (4) and that in the mobility matrix $\mathcal{M}^{\infty}$ are the same. With the grand mobility matrix used in Stokesian dynamics that includes forces, torques, and stresslets, the inversion takes into account all reflections from these force moments among all particles.

The convergence of the method of reflections is investigated by comparing the force calculated by the method of

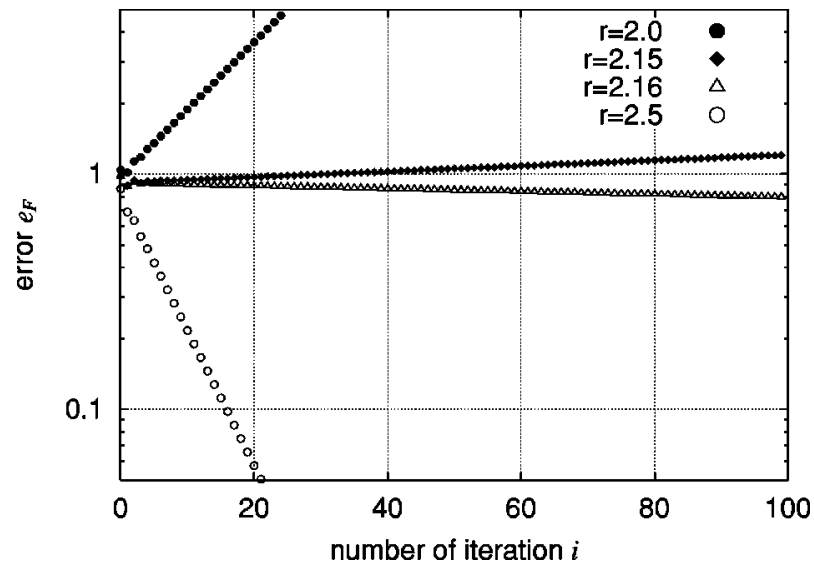

FIG. 2. Error $e_{F}$ of forces $F^{(i)}$ with number of iterations $i$ by the method of reflections for a three-body problem with a triangular configuration (10) with separations $r=2.0,2.15,2.16$, and 2.5 and velocities (11).

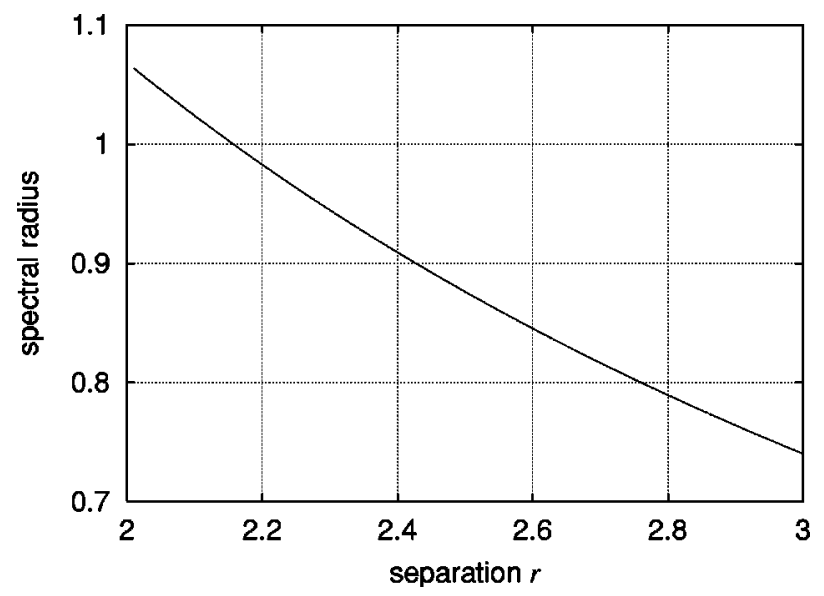

FIG. 3. Spectral radius of the iteration matrix " $A_{2} \cdot\left(A_{1}\right)^{-1}$, for the triangle configuration (10) with separation $r$. The spectral radius exceeds unity for $r<2.16$.

reflections, $\mathbf{F}^{(i)}$ in (4), with the expected value obtained by the inverse of the mobility matrix, $\mathbf{F}^{\infty}$ in (9). For a two-body problem, the method of reflections was found to converge for all particle separations. With three or more particles this was not the case.

Consider a three-body problem where the configuration and velocities are given by

$$
\begin{aligned}
& \mathbf{x}_{1}=\left[\begin{array}{c}
0 \\
r / 2 \\
0
\end{array}\right], \quad \mathbf{x}_{2}=\left[\begin{array}{c}
0 \\
-r / 2 \\
0
\end{array}\right], \quad \mathbf{x}_{3}=\left[\begin{array}{c}
\sqrt{\frac{3}{2}} r \\
0 \\
0
\end{array}\right], \\
& \mathbf{U}_{1}=\mathbf{U}_{2}=\mathbf{U}_{3}=\left[\begin{array}{l}
1 \\
0 \\
0
\end{array}\right],
\end{aligned}
$$

corresponding to particles arranged at the corners of an equilateral triangle. The particle radii have been set to unity. Figure 1 shows several values of the $x$-component of the force on the third particle $\left(F_{3}\right)_{x}$ calculated by (9) and (4) with $i=0,2,4$, and 6 . In this case, the method of reflections converges only for large separations $r \geqslant 2.16$. Figure 2 shows the error in the force $\mathbf{F}^{(i)}$ defined by

$$
e_{F}=\left|\mathbf{F}^{(i)}-\mathbf{F}^{\infty}\right| /\left|\mathbf{F}^{\infty}\right| .
$$

We see that $e_{F}$ increases with the number of iterations for $r<2.16$. Figure 3 shows the variation of the spectral radius of the iteration matrix " $A_{2} \cdot\left(A_{1}\right)^{-1}$,, with separation $r$. The critical distance $r_{c}$ where the spectral radius is equal to unity is $r_{c}=2.1567$ and agrees with the point where the method of reflections fails to converge.

There are several comments on this breakdown. This three-body configuration was also calculated previously by a variant of the method of reflections ${ }^{2,9}$ and the divergence was reported as "numerical instability." We believe that the divergence is due to the breakdown presented here. If we consider motion perpendicular to the plane of the particles rather than parallel to it, then the method of reflections might converge for all separations. This is because the eigenvalue that exceeds unity is related to parallel motion. Note that the 
TABLE I. Critical distance $r_{c}$ with number of particles $N$. Because $r_{c}<2$, the method of reflections with $N=2$ always converge.

\begin{tabular}{cccccc}
\hline \hline & \multicolumn{5}{c}{$N$} \\
& 2 & 3 & 4 & 5 & 6 \\
\hline$r_{c}$ & 1.1369 & 2.1567 & 2.9924 & 3.5978 & 4.0744 \\
\hline \hline
\end{tabular}

maximum eigenvalue is set by the geometry of the particle configuration, but certain specific motions may not couple to this eigenvalue. The breakdown is not specific to the configuration (10), however. In fact, Table I shows the critical distance $r_{c}$ for configurations where particles are placed at the corners of regular polygons with the number of particles $N=2,3$ (equilateral triangle), 4 (square), 5 (pentagon), and 6 (hexagon); $r_{c}$ increases with $N$.

The convergence failure is not due to the low order of the multipole truncation. We also calculated $r_{c}$ with higher truncations in terms of a general mobility problem relating irreducible velocity moments and irreducible force moments. ${ }^{5}$ The order of truncation $p$ is the maximum order of moments that are taken into account; F and FTS versions correspond to $p=0$ and 1 , respectively. Table II shows that $r_{c}$ increases with $p$, and therefore higher moments do not help the convergence. Although the critical separation becomes larger as $p$ increases, the rate of convergence of $\mathbf{F}^{(i)}$ is almost the same (slightly worse) with increasing $p$. Evidently, the largest eigenvalue for the parallel motion is independent of $p$. The reason for the breakdown is that the single-body solution is no longer a good approximation to $\mathcal{M}^{\infty}$, especially for small $r$ and large $N$.

The breakdown of the method of reflections can be overcome if we use conjugate-gradient-type iterative methods, which are widely used for sparse matrix problems. ${ }^{8}$ Here, we use the generalized minimum residual method (GMRES) $)^{10}$ for the $\mathrm{F}$ version since $\mathcal{M}^{\infty}$ is symmetric. Very accurate values with errors of $O\left(10^{-16}\right)$ can be obtained for all cases considered above with only two iterations. It is worth noting that even in the case of large separations the convergence of the method of reflections can be poor; for example, the error for a separation $r=2.5$ with (10) and (11) is $O\left(10^{-6}\right)$ at the 100th iteration.

We have shown that the method of reflections in resistance form with truncated multipoles is equivalent to the inversion of the mobility matrix with the same truncation. In turn, this means that the inversion of the mobility matrix takes into account the many-body effects associated with an infinite series of reflected interactions among all particles. Although the method of reflections in mobility form has been proven to converge, ${ }^{4}$ in resistance form the method may fail

TABLE II. Critical distance $r_{c}$ with order of truncation $p$. The truncation with $p=0$ and $p=1$ corresponds to $\mathrm{F}$ and FTS versions, respectively.

\begin{tabular}{|c|c|c|c|c|c|c|}
\hline & & & $p$ & & & \\
\hline & $0(\mathrm{~F})$ & $1(\mathrm{FTS})$ & 2 & 3 & 4 & 5 \\
\hline$r_{c}$ & 2.1567 & 2.5585 & 2.6344 & 2.6821 & 2.7140 & 2.7556 \\
\hline
\end{tabular}

to converge, especially for close configurations and for systems with many particles. Physically, this breakdown arises because the method is based on the assumption that the single-body solution is a good initial guess. For a group of near-touching particles moving in the same direction, the total force on the cluster will scale with the radius of the sphere that encloses the cluster, $R$, and thus the force on each individual particle scales with $R / N$, rather than with the particle size $a$. As $N$ grows the single particle solution becomes a poorer and poorer initial guess to the correct force behavior. Fortunately, however, alternate, conjugate-gradient-type iterative methods work well in cases where the method of reflections fails.

The application of iterative methods to determine particle interactions in Stokes flow could provide a substantial improvement for numerical calculations with large numbers of particles. In conventional Stokesian dynamics the resistance matrix for a many-body system is approximated by

$$
\mathcal{R}=\left(\mathcal{M}^{\infty}\right)^{-1}+\mathcal{R}^{\text {lub }},
$$

where $\mathcal{R}^{\text {lub }}$ is the near-field lubrication interactions constructed from the exact solution of two near-touching particles. Because of the matrix inversion of $\mathcal{M}^{\infty}$, the computational load of this method is very large and limits the size of the systems that can be studied. If we do not need to obtain the resistance matrix explicitly, but rather only the forces exerted on the particles, as is often the case, then the procedure to evaluate the forces would be improved computationally by applying an iterative method. ${ }^{5}$ As shown earlier in this paper, the method of reflections is not a good choice because of its slow, and loss of, convergence, but iterative methods like GMRES for $\mathrm{F}$ version and BiCGSTAB ${ }^{11}$ for higher truncated versions with nonsymmetric matrices are superior and converge.

\section{ACKNOWLEDGMENTS}

This work was supported by the Japan Society for the Promotion of Science and by funds from Kodak.

${ }^{1}$ J. Happel and H. Brenner, Low Reynolds Number Hydrodynamics (Martunus Nihhoff, Dordrecht, 1973).

${ }^{2}$ S. Kim and S. J. Karrila, Microhydrodynamics (Butterworth-Heinemann, Boston, 1991).

${ }^{3}$ L. Durlofsky, J. F. Brady, and G. Bossis, “Dynamic simulation of hydrodynamically interacting particles,' J. Fluid Mech. 180, 21 (1987).

${ }^{4}$ J. H. C. Luke, "Convergence of a multiple reflection method for calculating Stokes flow in a suspension,' SIAM (Soc. Ind. Appl. Math.) J. Appl. Math. 49, 1635 (1989).

${ }^{5} \mathrm{~K}$. Ichiki, "Improvement of the Stokesian Dynamics method for systems with a finite number of particles,' J. Fluid Mech. (submitted).

${ }^{6}$ J. F. Brady and G. Bossis, "Stokesian Dynamics,', Annu. Rev. Fluid Mech. 20, 111 (1988).

${ }^{7}$ C. G. Jacobi, Astron. Nachr. 22, 297 (1845).

${ }^{8}$ R. Weiss, Parameter-Free Iterative Linear Solvers (Akademie Verlag, Berlin, 1996).

${ }^{9}$ S. Kim, "Stokes flow past three spheres: An analytic solution,' Phys. Fluids 30, 2309 (1987).

${ }^{10}$ Y. Saad and M. H. Schultz, "GMRES: A generalized minimal residual algorithm for solving nonsymmetric linear systems," SIAM (Soc. Ind. Appl. Math.) J. Sci. Stat. Comput. 7, 856 (1986).

${ }^{11}$ H. A. van der Vorst, "BI-CGSTAB: A fast and smoothly converging variant of BI-CG for the solution of nonsymmetric linear systems," SIAM (Soc. Ind. Appl. Math.) J. Sci. Stat. Comput. 13, 631 (1992). 